\title{
Normalization of CA19-9 Following Resection for Pancreatic Ductal Adenocarcinoma is not Tantamount to being Cured?
}

\author{
Tao Chen ${ }^{1 \& *}$, Min-Gui Zhang ${ }^{2 \&}$, Xian-Jun Yü ${ }^{3 \&}$ Liang Liu ${ }^{3 \&}$
}

\begin{abstract}
Background: Postoperative carbohydrate antigen 19-9 (CA19-9) is an independent predictor of survival for pancreatic ductal adenocarcinoma (PDAC), and more powerful than preoperative CA19-9. However, making decisions just dependent on postoperative CA19-9 may result in necessary treatments not being performed. Materials and Methods: A total of $\mathbf{1 7 8}$ patients with resected PDAC were eligible for this retrospective study, classified into two corresponding subgroups according to postoperative CA19-9. Prognostic significance of all clinicopathologic factors was evaluated by univariate and multivariate analyses. Results: Postoperative CA19-9, preoperative $\mathrm{CA125}$ and lymph node status were independent predictors. Better predictive performances for overall survival (OS) and recurrence-free survival (RFS) were achieved by postoperative CA19-9 compared to preoperative CA125 and lymph node status. Particularly, preoperative CA125 was associated with poor OS $(p<0.001$ for the normalized CA19-9 patients, $p=0.012$ for the elevated) and RFS ( $p=0.005$ for the normalized, $p=0.004$ for the elevated). Moreover, preoperative $\mathrm{CA125}$ levels related with survival in double- negative patients. Conclusions: Normalization of CA19-9 is not tantamount to be cured. Preoperative CA125 is a critical predictor for PDAC patients, especially in double-negative patients.
\end{abstract}

Keywords: Postoperative CA19-9 - normalization - preoperative CA125 - pancreatic ductal cancer - prognosis

Asian Pac J Cancer Prev, 16 (2), 661-666

\section{Introduction}

Although surgical management and adjuvant therapy develop rapidly, the prognosis of patients with resectable pancreatic ductal adenocarcinoma (PDAC) remains poor. Five-year survival rate was just $20 \%$ in the era of adjuvant chemo- or chemoradiotherapy (Siegel et al., 2013). So, it is particularly crucial to predict prognosis and guide postoperative treatment, which highlights the importance of adjuvant therapy.

Many serum markers are connection with PDAC, including serum carbohydrate antigen 19-9 (CA19-9), CA125, carcinoembryonic antigen (CEA) (Tempero et al., 2010; Liu et al., 2014; Zhang et al., 2014). Perioperative serum CA19-9 is the most widely used in the diagnosis, prognosis, and remains representative to monitor recurrence and metastasis after undergoing curative resection (Ferrone et al., 2006; Castellanos, 2014). Preoperative CA19-9 focuses on diagnosis, and postoperative CA19-9 corresponds to tumor recurrence after surgery. As always, baseline preoperative elevated serum CA19-9 levels indicate dismal prognosis in series of retrospective analyzes in PDAC (Lim et al., 2003; Schlieman et al., 2003; Ferrone et al., 2006). Recently, the role of postoperative CA19-9 levels was gradually recognized. Postoperative serum CA19-9, but not preoperative CA19-9, significantly predicted the survival of PDAC patients (Ferrone et al., 2006; Kondo et al., 2010; Motoi et al., 2011;Hata et al., 2012; Tzeng et al., 2013). Failure of normalization of CA19-9 after resection for PDAC is tantamount to metastatic disease and adverse prognosis (Abdel-Misih et al., 2011). Dose normalization of CA19-9 following resection mean to be cured? The answer is not absolute. Some patients with slightly elevated postoperative CA19-9 have an excellent prognosis. Similarly, normalized postoperative CA19-9 is not tantamount to be cured. Therefore, it does not well evaluate the prognosis by postoperative CA19-9 alone, especially in normalized patients.

CA125, a high-molecular-weight mucin-like glycoprotein over-expressed in ovarian cancer and other cancers, is the most useful serologic marker for ovarian cancer detection and management (Schmidt, 2011; Tse et al., 2014). The prognostic value of CA125 had been described in previous studies (Liu et al., 2014). Importantly, it is not influenced by serum bilirubin (Haglund, 1986). In clinical work, perioperative CA19-9 and pathologic correlation parameters (presence of lymph node metastasis, TNM stages), as independent indictors, are widely used in adjustment of chemotherapy therapy (NCCN). This study focused on the prognostic impact of clinicopathologic factors, including serum CA125

${ }^{1}$ Department of Surgery, Huadong Hospital Affiliated to Fudan University, ${ }^{2}$ Department of Ophthalmology, Shanghai Tenth People's Hospital, Tongji University School of Medicine, ${ }^{3}$ Department of Pancreas and Hepatobiliary Surgery, Fudan University, Shanghai Cancer Center, Shanghai, China ${ }^{\circledR}$ Equal contributors *For correspondence: zhejiangchentao@163.com 
level, pathologic parameters in PDAC patients following macroscopically curative surgical resection based on postoperative serum CA19-9.

\section{Materials and Methods}

\section{Patient cohort and data collection}

From March 2010 to May 2012, a total of 178 consecutive PDAC patients who underwent surgical resection were enrolled in this retrospective study at Department of Pancreas and Hepatobiliary Surgery, Fudan University, Shanghai Cancer Center. Patients with data on perioperative CA125, and postoperative CA19-9 levels were selected to investigate the role of these tumor markers in the prediction of prognosis. Preoperative serum levels of CA19-9 and CA125 were simultaneously measured within 14 days before surgery, and postoperative CA199 and CA125 were defined as the lowest levels within 3 to 4 months after resection. The normal upper limit of CA19-9 is $37 \mathrm{U} / \mathrm{ml}$ (Kondo et al., 2010; Motoi et al., 2011). Nonsecretors (CA 19-9 <5 U/ml) were excluded from this study. Other variables were also evaluated, included age, gender, tumor location, tumor size, type of resection, adjuvant chemotherapy, tumor differentiation, TNM stages, and presence of lymph node.

The study was approved by the Shanghai Cancer Center Research Ethics Committee. Informed consent was

Table 1. Clinicopathological Features

\begin{tabular}{lc}
\hline Features & Values/Counts $(\mathrm{n}=178)$ \\
\hline Age (years, median (range)) & $61(33-82)$ \\
Gender (male/female) & $108 / 70$ \\
Tumor location (head/body, tail) & $114 / 64$ \\
Tumor size (cm, mean \pm SD) & $4.3 \pm 1.5$ \\
Lymph node metastasis (yes/no) & $77 / 101$ \\
Tumor differentiation (I,II/III) & $139 / 39$ \\
Neural invasion (yes/no) & $14 / 31$ \\
Microvascular invasion (yes/no) & $31 / 147$ \\
Preoperative CA 125 & 133.0 \\
(U/mL, median (range)) & $(2.57 \geq 251.50)$ \\
Postoperative CA 19-9 & 22.1 \\
(U/mL, median (range)) & $(0.8 \geq 2111.0)$ \\
T stage (I/II/III) & $0 / 42 / 147$ \\
TNM stage (IB/IIA/IIB) & $23 / 78 / 88$ \\
\hline
\end{tabular}

obtained from each patient according to the committee's regulations.

\section{Follow-up}

In the first year, serologic examination was done every month, and CT of the abdomen was done every 3.0 months. If recurrence was suspected, a CT, MRI or $\mathrm{PET} / \mathrm{CT}$ was done immediately. Overall survival (OS) was defined as the time from the dates of surgery to death or last follow-up. Recurrence-free survival (RFS) was

Table 2 Relations between the Parameters and Pathological Features

\begin{tabular}{|c|c|}
\hline Postoperative CA19-9 & Preoperative CA125 \\
\hline $\begin{array}{cc}\text { Low } & \text { High } p \text { value } \\
(\mathrm{n}=117) & (\mathrm{n}=72)\end{array}$ & $\begin{array}{l}\text { Low } \\
(\mathrm{n}=96)(\mathrm{n}=82)\end{array}$ \\
\hline
\end{tabular}

\begin{tabular}{|c|c|c|c|c|c|c|}
\hline \multicolumn{7}{|l|}{ Age } \\
\hline$\leq 61$ year & 58 & 29 & 0.306 & 46 & 40 & 0.741 \\
\hline$>61$ year & 59 & 43 & & 50 & 42 & \\
\hline \multicolumn{7}{|l|}{ Gender } \\
\hline Female & 48 & 33 & 0.523 & 40 & 30 & 0.492 \\
\hline Male & 69 & 39 & & 56 & 52 & \\
\hline \multicolumn{7}{|l|}{ Tumor size } \\
\hline$\leq 3 \mathrm{~cm}$ & 48 & 24 & 0.213 & 32 & 31 & 0.314 \\
\hline$>3 \mathrm{~cm}$ & 69 & 48 & & 64 & 51 & \\
\hline \multicolumn{7}{|c|}{ Lymph node metastasis } \\
\hline No & 74 & 38 & 0.015 & 55 & 46 & 0.874 \\
\hline Yes & 43 & 34 & & 41 & 36 & \\
\hline \multicolumn{7}{|l|}{ Neural invasion } \\
\hline No & 20 & 12 & 0.849 & 21 & 10 & 0.199 \\
\hline Yes & 9 & 60 & & 75 & 72 & \\
\hline \multicolumn{7}{|c|}{ Microvascular invasion } \\
\hline No & 101 & 56 & 0.069 & 79 & 68 & 0.735 \\
\hline Yes & 16 & 16 & & 17 & 14 & \\
\hline \multicolumn{7}{|c|}{ Tumor differentiation } \\
\hline Poorly & 92 & 58 & 0.810 & 72 & 68 & 0.283 \\
\hline Well/Moderately & 25 & 14 & & 24 & 14 & \\
\hline \multicolumn{7}{|c|}{ T stage } \\
\hline $\mathrm{T} 1$ & 0 & 0 & 0.376 & 0 & 0 & 0.125 \\
\hline $\mathrm{T} 2$ & 30 & 11 & & 27 & 15 & \\
\hline $\mathrm{T} 3$ & 87 & 61 & & 69 & 67 & \\
\hline \multicolumn{7}{|l|}{ TNM stage } \\
\hline IB & 19 & 4 & 0.008 & 16 & 7 & 0.487 \\
\hline IIA & 55 & 34 & & 39 & 39 & \\
\hline IIB & 43 & 34 & & 41 & 36 & \\
\hline
\end{tabular}

Table 3. Univariate and Multivariate Analyses of Factors Associated With Survival and Recurrence

\begin{tabular}{|c|c|c|c|c|c|c|c|c|}
\hline \multirow[t]{3}{*}{ Variables } & \multicolumn{4}{|c|}{ Overall Survival } & \multicolumn{4}{|c|}{ Time to Recurrence } \\
\hline & \multirow{2}{*}{$\begin{array}{c}\text { Univariate } \\
\text { P value }\end{array}$} & \multicolumn{3}{|c|}{ Mutivariate } & \multirow{2}{*}{$\begin{array}{c}\text { Univariate } \\
p \text { value }\end{array}$} & \multicolumn{3}{|c|}{ Mutivariate } \\
\hline & & HR & $95 \% \mathrm{CI}$ & $\overline{p \text { value }}$ & & $\overline{\mathrm{HR}}$ & $95 \% \mathrm{CI}$ & $p$ value \\
\hline Age $(\leq 61$ year / >61 year) & 0.492 & NA & NA & NA & 0.560 & NA & NA & NA \\
\hline Gender (male/female) & 0.372 & NA & NA & NA & 0.284 & NA & NA & NA \\
\hline Tumor location (head/body, tail) & 0.307 & NA & NA & NA & 0.453 & NA & NA & NA \\
\hline Tumor size $(\leq 3 \mathrm{~cm} />3 \mathrm{~cm})$ & 0.246 & NA & NA & NA & 0.517 & NA & NA & NA \\
\hline Lymph node metastasis (yes/no) & 0.001 & 1.670 & $1.117-2.495$ & 0.012 & 0.001 & 1.826 & $1.271-2.623$ & 0.001 \\
\hline Tumor differentiation (I/II/III) & 0.709 & NA & NA & NA & 0.068 & NA & NA & NA \\
\hline Neural invasion (yes/no) & 0.283 & NA & NA & NA & 0.157 & NA & NA & NA \\
\hline Microvascular invasion (yes/no) & 0.951 & NA & NA & NA & 0.425 & NA & NA & NA \\
\hline TNM stage (IB/IIA/IIB) & 0.008 & 1.391 & $1.001-1.933$ & 0.049 & 0.003 & 1.490 & $1.111-1.999$ & 0.008 \\
\hline T stage (I/II/III) & 0.936 & NA & NA & NA & 0.947 & NA & NA & NA \\
\hline Postoperative CA19-9 ( $\leq 37 \mathrm{U} / \mathrm{mL} />37 \mathrm{U} / \mathrm{mL})$ & $<0.001$ & 1.941 & $1.288-2.927$ & 0.002 & $<0.001$ & 2.679 & $1.861-3.856$ & $<0.001$ \\
\hline Chemotherapy (yes/no) & 0.010 & 0.421 & $0.222-0.799$ & 0.008 & 0.035 & 0.0529 & $0.292-0.956$ & 0.035 \\
\hline Preoperative CA125 $(\leq 18.6 \mathrm{U} / \mathrm{mL} />18.6 \mathrm{U} / \mathrm{mL})$ & $<0.001$ & 2.391 & $1.600-3.572$ & $<0.001$ & $<0.001$ & 2.096 & $1.454-3.020$ & $<0.001$ \\
\hline
\end{tabular}


Normalization of CA19-9 Following Resection for Pancreatic Ductal Adenocarcinoma is not Tantamount to being Cured?

defined as the interval between the dates of surgery and tumor recurrence or the last follow up.

\section{Statistical analysis}

Statistical Package for Social Science (SPSS) version 16.0 for Windows was used for statistical analysis. The quantitative variables age, follow-up time, serum levels of CA19-9 and CA125, were expressed as medians with interquartile ranges. Survival curves were calculated by using the Kaplan-Meier method and log rank tests for clinical and pathological factors. Factors found to be significant by univariate statistical analysis were entered into a multivariate analysis (Cox regression) to determine independent prognostic factors. Receiver operating characteristics (ROC) curves were used to evaluate the predictive performance for independent prognostic factors. In order to better evaluate the prognostic values, patients were divided according to postoperative CA19-9 and lymph node metastasis. The relation between CA125 and prognosis was also calculated. Two-sided $\mathrm{P}$ values were considered statistically significant at $p<0.05$.

\section{Results}

\section{Demographics and outcome}

There were 178 patients had perioperative CA19-9 and CA125 values documented. There were 119 (66.4\%) were men and $70(33.6 \%)$ were women, the median age was 61 years (range 33 to 82 years). One hundred and eight patients $(60.6 \%)$ underwent pancreaticoduodenectomy or total pancreatectom, and 64 (39.4\%) underwent distal pancreatectomies. Of these patients, 77 (43.3\%) had regional lymph node metastasis, and 164 patients received postoperatively adjuvant chemotherapy. In general, those patients underwent standard gemcitabine-based chemotherapy, and considered for second-line therapy that demonstrated recurrence or progression. Recurrence occurred in 124 patients $(69.7 \%)$, overall survival rates at 1,2 , and 3 years were $91.6 \%, 60.1 \%$, and $32.4 \%$, and the median survival time was 19.7 (range 6.3 to 60.6 ) months. The demographic and clinical characteristics of the patients were list in Table 1 .

A strong positive correlation was observed between postoperative CA19-9 and presence of lymph node metastasis $(\mathrm{r}=0.182, p=0.015)$, and TNM stage $(\mathrm{r}=0.198$, $p=0.008)$. There were no statistically significant differences between postoperative CA19-9 or preoperative CA125 and other clinicopathological characteristics, such as tumor location, differentiation, microvascular invasion, neural invasion, or tumor size (Table 2).

\section{Univariate and multivariate analysis for survival}

To evaluate the predictive value of all above parameters, univariate and multivariate models were performed. The univariate analysis indicated that high postoperative CA19-9 and high preoperative high CA125 were statistically significant risk predictors of poor OS (both $p<0.001$ ) and RFS (both $p<0.001$ ), in addition to lymph node metastasis (both $p=0.001$ ), high TNM stages and postoperatively adjuvant chemotherapy (Fig 1). There was no significant relation between other clinicopathological indictors and either OS or RFS. Postoperative CA19$9(p=0.002, \mathrm{HR}=1.941,95 \% \mathrm{CI}, 1.288-2.927$ for OS; $p<0.001, \mathrm{HR}=2.679,95 \% \mathrm{CI}, 1.861-3.856$ for $\mathrm{RFS}$; respectively), preoperative $\mathrm{CA} 125(p<0.001, \mathrm{HR}=2.391$, 95\% CI, 1.600-3.572 for OS; $p<0.001, \mathrm{HR}=2.096$, 95\%CI, 1.454-3.020 for RFS, respectively), lymph node metastasis $(p=0.012, \mathrm{HR}=1.670,95 \% \mathrm{CI}, 1.117-2.495$ for OS; $p=0.001, \mathrm{HR}=1.826,95 \% \mathrm{CI}, 1.271-2.623$ for RFS; respectively), TNM stages, and postoperative chemotherapy were independent predictors of poor survival in multivariate analysis (Table 3 ).

Better predictive performances for OS and RFS were achieved by postoperative CA19-9 (AUC=0.612 for OS, $\mathrm{AUC}=0.666$ for RFS) compared to preoperative CA125 (AUC $=0.610$ for $\mathrm{OS}, \mathrm{AUC}=0.591$ for RFS), lymph node status (AUC $=0.597$ for OS, AUC 0.598 for RFS), TNM stages $(\mathrm{AUC}=0.606$ for $\mathrm{OS}, \mathrm{AUC}=0.608$ for $\mathrm{RFS}$ ), and chemotherapy (AUC $=0.533$ for $\mathrm{OS}, \mathrm{AUC}=0.543$ for $\mathrm{RFS}$, respectively) by ROC analysis.

Survival analysis in subgroups divided by postoperative CA19-9 orland lymph node metastasis

In the subgroups divided by postoperative CA19-9, preoperative CA125 was a significant prognostic factor for both OS ( $p<0.001$ for the normalized, $p=0.012$ for the elevated, respectively) and RFS ( $p=0.005$ for the normalized, $p=0.004$ for the elevated, respectively), whereas lymph node status ( $p=0.041$ for OS, $p=0.080$ for RFS, respectively) was significant in the normalized only.
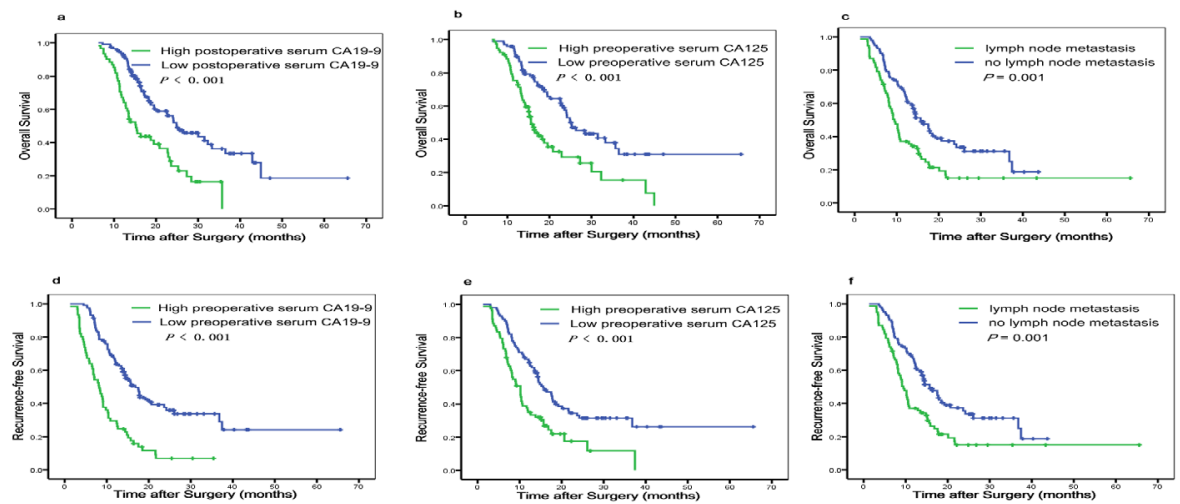

Figure 1. Kaplan-Meier Analysis for Survival. (A,D): Kaplan-Meier survival curves for OS and RFS based on postoperative CA19-9 (both $p<0.001$ ); (B, E): Kaplan-Meier survival curves for OS and RFS based on preoperative CA125 (both $p<0.001$ ); (C, F): Kaplan-Meier survival curves for OS and RFS based on lymph node metastasis (both $p=0.001$ ) 

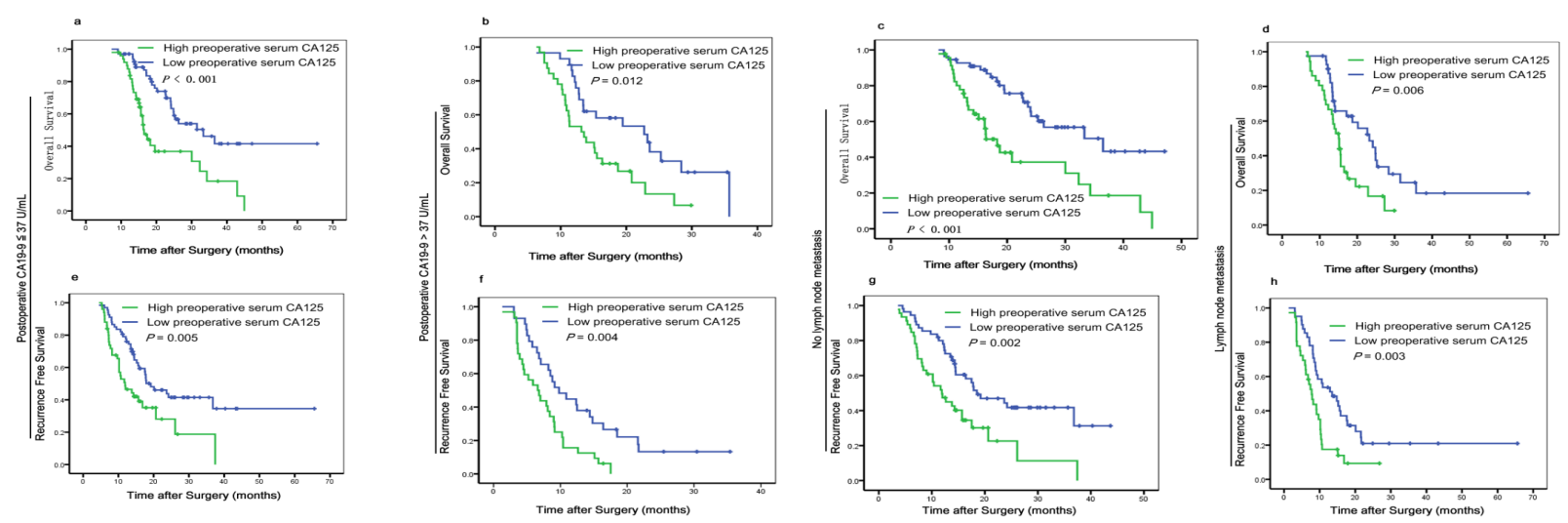

Figure 2. Kaplan-meier Analysis of Survival in the Subgroups Divided by Postoperative CA19-9 or Lymph Node Metastasis. Preoperative CA125 was a significant prognostic factor for both OS $(p<0.001$ for the normalized, $p=0.012$ for the elevated, respectively) (A, B), and RFS ( $p=0.005$ for the normalized, $p=0.004$ for the elevated, respectively) (E, F). The same conclusion was drawn in subgroup divided by lymph node metastasis $(\mathbf{C}, \mathbf{D}, \mathbf{G}, \mathbf{H})$
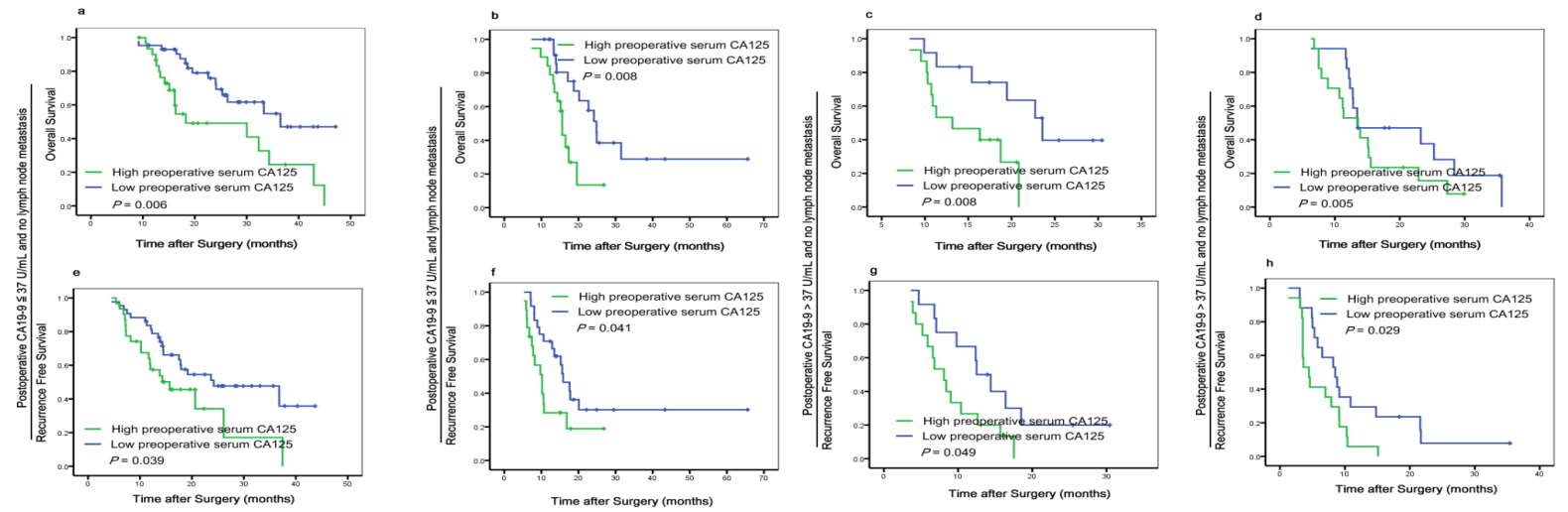

Figure 3. Kaplan-Meier Analysis of Survival in Subgroups by Postoperative CA19-9 and Lymph Node Metastasis

Similarly, preoperative CA125 was related with patient survival (no lymph node metastasis patients: $p<0.001$ for OS, $p=0.002$ for RFS; lymph node metastasis patients: $p=0.006$ for OS, $p=0.003$ for RFS, respectively) (Figure 2).

To further eliminate the effect from lymph nodes, subgroup analysis according to postoperative CA19-9 and lymph nodes status showed that preoperative CA 125 was also a risk factor for OS ( $p=0.008$ for negative lymph nodes patients with elevated CA19-9, $p=0.008$ for lymph nodes metastasis patients with normalized CA19-9, respectively) and RFS ( $p=0.041$ for negative lymph nodes patients with elevated CA19-9, $p=0.049$ for lymph nodes metastasis patients with normalized CA19-9, respectively) (Figure 3). What is worth mentioning is that CA125 is related with survival ( $p=0.006$ for OS, $p=0.039$ for RFS, respectively) in the double-negative patients, regardless of lymph node status and postoperative CA19-9.

\section{Discussion}

Because of microscopic metastatic disease occult at time of surgery, PDAC portends dismal prognosis even in patients with surgical resection. Therefore, an accurate prediction of the prognosis makes a timely treatment for patients and improves their prognosis. Serum preoperative CA19-9 levels reflect tumor burden and related with patient survival (Ferrone et al., 2006; Xu et al., 2014). Clinicians generally utilize perioperative CA19-9 to monitor tumor recurrence and guide treatment strategies. However, CA19-9 is not a perfect predictive marker: hyperbilirubinaemia induces an increase in serum CA19-9 levels (Katz et al., 2010), and the nearly $30 \%$ of patients did not show an increase in CA19-9 levels (Schlieman et al., 2003). In previous retrospective studies, hyperbilirubinaemia and nonsecretors were excluded from their analysis (Ferrone et al., 2006; Motoi et al., 2011). Recently, clinicians begin to focus on the prognostic value of postoperative CA19-9, which had been demonstrated as an independent predictor of survival, and a better prognostic marker than preoperative CA19-9 (Ferrone et al., 2006; Barugola et al., 2009; Kondo et al., 2010; Abdel-Misih et al., 2011; Hata et al., 2012; Humphris et al., 2012; Hartwig et al., 2013). Because of no postoperative jaundice and short half-life period of serum CA19-9 after surgery (Rapellino et al., 1994), this indictor escapes from the interference of hyperbilirubinaemia and preoperative CA19-9. Previous reports revealed that persistent CA199 elevation after resection was analogous to metastatic disease and the elevated had significantly poorer survival in comparison with the normalized ones. In fact, some normalized CA19-9 PDAC patients also had a poor prognosis, and even worse than those elevated patients. This phenomenon is also worth considering. Here, we showed preoperative serum CA125 had a distinct potential utility in PDAC patients, when assessed at distinct clinical decision follow surgical resection.

Serum CA125 had been proved as a potential predictor for PDAC survival (Haglund, 1986; Cwik et al., 2006; Liu 
Normalization of CA19-9 Following Resection for Pancreatic Ductal Adenocarcinoma is not Tantamount to being Cured?

et al., 2014), and related with carcinomatosis in pancreatic cancer and other cancers (may partly originate from the peritoneum in patients with metastatic disease) (Haridas et al., 2011; Chen et al., 2012; Emoto et al., 2012; Wagner et al., 2013). We have been demonstrated that "triple positive PDAC patients" (CEA+/CA125+/CA19-9 $\geq$ $1,000 \mathrm{U} / \mathrm{mL}$ ) had a poor prognosis following resection (Liu et al., 2014). More importantly, serum CA125 levels were not interfered by serum bilirubin levels (Haglund, 1986). In this retrospective analysis, postoperative CA199, preoperative CA125, and presence of lymph node metastasis were independent prognosticators for both OS and RFS after adjusting for age, sex, tumor size, location, differentiation, neural invasion, and microvascular invasion. And the prognostic value of postoperative CA19-9 was priorior to perioperative CA125, preoperative CA19-9 and presence of lymph node metastasis by ROC curve, which coincides with the present studies (AbdelMisih et al., 2011).

In order to better predict prognosis and guide treatments, patients were divided into two subgroups by postoperative CA19-9 levels: the normalized and the elevated. Those patients with high preoperative CA125 had shorter median OS times (the normalized: median survival time 23.1 vs 40.5 months, $p<0.001$; the elevated: OS 15.2 vs 22.1 months, $p=0.012$ ) and RFS times (the normalized: 17.1 vs 33.2 months, $p=0.005$; the elevated: 7.5 vs 13.7 months, $p=0.004$ ) than patients with low CA125. And similar results were found in patients with positive lymph nodes, but not for postoperative CA125.

To get an in-depth research, patients were stratified into four groups according for postoperative CA199 and lymph nodal status, and the prognostic value of preoperative CA125 was also assessed. Arguably, preoperative CA125 levels related with survival in doublenegative patients (median survival time 25.9 vs 34.8 months, $p=0.006$ for OS; 18.6 vs 27.4 months, $p=0.039$ for RFS, respectively), lymph node metastasis patients with normalized CA19-9 (median survival time 16.4 vs 33.9 months, $\mathrm{P}=0.008$ for OS; 12.4 vs 28.8 months, $p=0.041$ for RFS, respectively) and no lymph node metastasis patients with elevated CA19-9 (median survival time 14.8 vs 22.9 months, $p=0.017$ for OS; 9.1 vs 15.4 months, $p=0.049$ for RFS, respectively). These results might cause by micro metastases disease at the time of surgery and postoperative timely adjuvant chemotherapy. In patients with lymph node metastasis, postoperative adjuvant chemotherapy maybe induce a decrease in serum CA19-9 levels. It may be also caused by distance undetected metastases disease, in addition to lymph node metastasis. For no lymph node metastasis patients with elevated postoperative CA19-9 levels, incomplete lymph node dissection, skip metastasis and preoperative micro metastases disease may lead to a poor prognosis. However, preoperative CA125 levels were not related with survival in the double-positive subgroup, which had the worst prognosis patients. Similarly, the postoperative CA125 was also evaluated its prognostic value by the same measure, no significant relationship with survival was confirmed.

Our study is inherently limited for general applications by its retrospective design. A large number of patients were excluded due to inadequate follow-up of postoperative CA19-9 levels. Additionally, serum CA125 and CA199 are not unique indicators of pancreatic cancer. For example, CA125 rises in response to cardiovascular (Kouris et al., 2005) and chronic liver disease (Miralles et al., 2003), endometriosis, and ovarian cancer (Longuespee et al., 2012). And colorectal cancer, gallbladder cancer, bile duct cancer, liver cancer and stomach cancer induce increase in CA19-9 levels. Finally, different surgeons have different lymph node dissections, which also lead to errors.

In conclusion, postoperative CA19-9 is an excellently prognostic indictor than preoperative CA19-9 and the presence of lymph node. However, normalization of postoperative CA19-9 for PDAC is not tantamount to be cured. Making decisions just dependent on postoperative CA19-9 may lose the necessary treatments or received excessive chemotherapy. This study suggests that it is necessary to bind postoperative CA19-9 with preoperative CA125 to design treatment strategies, especially for the normalized CA19-9 patients. Preoperative CA125 is related with survival in normalized and elevated CA19-9 patients.

\section{References}

Abdel-Misih SR, Hatzaras I, Schmidt C, et al (2011). Failure of normalization of CA19-9 following resection for pancreatic cancer is tantamount to metastatic disease. Ann Surg Oncol, 18, 1116-21.

Barugola G, Partelli S, Marcucci S, et al (2009). Resectable pancreatic cancer: who really benefits from resection? Ann Surg Oncol, 16, 3316-22.

Castellanos JA, Merchant NB (2014). Intensity of follow-up after pancreatic cancer resection. Ann Surg Oncol, 21, 747-51.

Chen SH, Dallas MR, Balzer EM, et al (2012). Mucin 16 is a functional selectin ligand on pancreatic cancer cells. FASEB $J, 26,1349-59$.

Cwik G, Wallner G, Skoczylas T, et al (2006). Cancer antigens $19-9$ and 125 in the differential diagnosis of pancreatic mass lesions. Arch Surg, 141, 968-73.

Emoto S, Ishigami H, Yamashita H, et al (2012). Clinical significance of CA125 and CA72-4 in gastric cancer with peritoneal dissemination. Gastric Cancer, 15, 154-61.

Ferrone CR, Finkelstein DM, Thayer SP, et al (2006). Perioperative CA19-9 levels can predict stage and survival in patients with resectable pancreatic adenocarcinoma. $J$ Clin Oncol, 24, 2897-902.

Haglund C (1986). Tumour marker antigen CA125 in pancreatic cancer: a comparison with CA19-9 and CEA. J Clin Oncol, 54, 897-901.

Haridas D, Chakraborty S, Ponnusamy MP, et al (2011). Pathobiological implications of MUC16 expression in pancreatic cancer. PloS one, 6, 26839.

Hartwig W, Strobel O, Hinz U, et al (2013). CA19-9 in potentially resectable pancreatic cancer: perspective to adjust surgical and perioperative therapy. Ann Surg Oncol, 20, 2188-96.

Hata S, Sakamoto Y, Yamamoto Y, et al (2012). Prognostic impact of postoperative serum CA 19-9 levels in patients with resectable pancreatic cancer. Ann Surg Oncol, 19, 636-41.

Humphris JL, Chang DK, Johns AL, et al (2012). The prognostic and predictive value of serum CA19.9 in pancreatic cancer. Ann Oncol, 23, 1713-22.

Katz MH, Varadhachary GR, Fleming JB, et al (2010). Serum CA 19-9 as a marker of resectability and survival in patients 


\section{Tao Chen et al}

with potentially resectable pancreatic cancer treated with neoadjuvant chemoradiation. Ann Surg Oncol, 17, 1794-801.

Kondo N, Murakami Y, Uemura K, et al (2010). Prognostic impact of perioperative serum CA 19-9 levels in patients with resectable pancreatic cancer. Ann Surg Oncol, 17, 2321-9.

Kouris NT, Zacharos ID, Kontogianni DD, et al (2005). The significance of CA125 levels in patients with chronic congestive heart failure. Correlation with clinical and echocardiographic parameters. Eur J Heart Fail, 7, 199-203.

Lim JE, Chien MW, Earle CC (2003). Prognostic factors following curative resection for pancreatic adenocarcinoma: a population-based, linked database analysis of 396 patients. Ann Surg, 237, 74-85.

Liu L, Xu H, Wang W, Wu C, et al (2014). A preoperative serum signature of CEA/CA125/CA19-9>/= $1000 \mathrm{U} / \mathrm{mL}$ indicates poor outcome to pancreatectomy for pancreatic cancer. Int $J$ Cancer. [Epub ahead of print]

Longuespee R, Boyon C, Desmons A, et al (2012). Ovarian cancer molecular pathology. Cancer Metastasis Rev, 31, 713-32.

Miralles C, Orea M, Espana P, et al (2003). Cancer antigen 125 associated with multiple benign and malignant pathologies. Ann Surg Oncol, 10, 150-4.

Motoi F, Rikiyama T, Katayose Y, et al (2011). Retrospective evaluation of the influence of postoperative tumor marker status on survival and patterns of recurrence after surgery for pancreatic cancer based on RECIST guidelines. Ann Surg Oncol, 18, 371-9.

Rapellino M, Piantino P, Pecchio F, et al (1994). Disappearance curves of tumor markers after radical surgery. Int J Biol Markers, 9, 33-7.

Schlieman MG, Ho HS, Bold RJ (2003). Utility of tumor markers in determining resectability of pancreatic cancer. Arch Surg, 138, 951-5

Schmidt C (2011). CA-125: a biomarker put to the test. J Natl Cancer Inst, 103, 1290-1.

Siegel R, Naishadham D, Jemal A (2013). Cancer statistics, 2013. CA Cancer J Clin, 63, 11-30.

Tempero MA, Arnoletti JP, Behrman S, et al (2010). Pancreatic adenocarcinoma. J Natl Compr Canc Netw, 8, 972-1017.

Tse BW, Collins A, Oehler MK, et al (2014). Antibody-based immunotherapy for ovarian cancer: where are we at? Ann Oncol, 25, 322-31.

Tzeng CW, Abbott DE, Cantor SB, et al (2013). Frequency and intensity of postoperative surveillance after curative treatment of pancreatic cancer: a cost-effectiveness analysis. Ann Surg Oncol, 20, 2197-203.

Wagner PL, Austin F, Sathaiah M, et al (2013). Significance of serum tumor marker levels in peritoneal carcinomatosis of appendiceal origin. Ann Surg Oncol, 20, 506-14.

Xu HX, Chen T, Wang WQ, et al (2014). Metabolic tumour burden assessed by (1)(8)F-FDG PET/CT associated with serum CA19-9 predicts pancreatic cancer outcome after resection. Eur J Nucl Med Mol Imaging, 41, 1093-102.

Zhang P, Zou M, Wen X, et al (2014). Development of serum parameters panels for the early detection of pancreatic cancer. Int J Cancer, 134, 2646-55. 